A N N A L E S Annales de Bretagne et des Pays de l'Ouest

\title{
Les moissons du ciel. 30 années d'archéologie aérienne au-dessus du massif armoricain
}

Daniel Pichot

\section{(2) OpenEdition}

Édition électronique

URL : https://journals.openedition.org/abpo/5626

DOI : $10.4000 / a b p o .5626$

ISBN : 978-2-7535-8091-6

ISSN : 2108-6443

Éditeur

Presses universitaires de Rennes

Édition imprimée

Date de publication : 30 juin 2020

Pagination : 183-186

ISBN : 978-2-7535-8090-9

ISSN : 0399-0826

\section{Référence électronique}

Daniel Pichot, «Les moissons du ciel. 30 années d'archéologie aérienne au-dessus du massif armoricain », Annales de Bretagne et des Pays de l'Ouest [En ligne], 127-2 | 2020, mis en ligne le 30 juin 2020, consulté le 04 janvier 2023. URL : http://journals.openedition.org/abpo/5626; DOI : https://doi.org/10.4000/ abpo.5626 


\section{Comptes-rendus}

Gautier, Maurice, Guigon, Philippe et Leroux, Gilles, Les moissons du ciel. 30 années d'archéologie aérienne au-dessus du massif armoricain, Rennes, Presses universitaires de Rennes, 2019, 431 p.

Avec ce gros et beau livre les trois auteurs atteignent à une brillante réussite en réunissant des qualités un peu paradoxales. Ses grandes dimensions, son poids et son format lui permettent d'accueillir plus de 400 illustrations en couleur et dans un format suffisant pour les apprécier, avec une mise en page agréable et efficace. Les trois associés dans cette aventure l'avouent dès leur introduction, ils ont voulu faire un livre pour les yeux et le parcourir constitue d'abord un plaisir. Cependant, ce n'est absolument pas la nième version d'un " Ouest vu du ciel " à parcourir d'un œil distrait mais, avant tout, un livre très sérieux et très savant : le bilan de trente années de recherche en archéologie aérienne au-dessus du Massif armoricain, une véritable somme. Pourtant, la lecture ne s'en révèle en rien fastidieuse car les trois auteurs nous livrent avec bonheur leur enthousiasme qu'ils nous font partager, non sans humour, ainsi que l'amitié qui les lie et soude leur équipe de travail. Ces 400 pages arrivent à allier science et passion pour le plus grand plaisir du lecteur. Le trio, fruit de l'alliance assez improbable au départ d'un ingénieur de l'Inrap dont les chantiers sont bien connus, d'un instituteur au palmarès archéologique très fourni, et d'un aiguilleur du ciel doté d'un doctorat en archéologie qui sert de pilote, nous livre aujourd'hui un bilan des 30 années d'une activité qui leur a fait survoler une bonne partie de l'Ouest chaque été.

Une première partie est consacrée à la présentation de la recherche et constitue une très bonne initiation à l'archéologie aérienne, sans lourdeur pédagogique, strictement orientée vers leur champ d'action qui ne va pas sans spécificités. Après avoir rendu hommage aux pionniers de la discipline et en particulier à R. Agache, le maître qui les a largement protégés et encouragés à leurs débuts, ils présentent les caractères et les contraintes de leur espace de travail : un Massif armoricain partagé avec d'autres ce qui leur réserve surtout le centre Bretagne et la haute Bretagne ainsi que les Pays de Loire.

A priori, la région ne se prêtait guère à la prospection aérienne : l'humidité du climat, les sols, et surtout le bocage dense ne permettaient guère, en théorie, une observation valable. En fait, les variations climatiques, la diversité des sols et surtout le massacre du bocage ont permis une observation inégale, certes, mais parfaitement possible. Il en résulte que la haute Bretagne et les Pays de Loire, moins humides et plus sujets aux contrastes climatiques, dotés souvent de sols plus favorables à l'observation présentent les meilleurs atouts.

Ensuite viennent les éléments essentiels de cette archéologie très particulière. D'abord, la recension des indices les plus révélateurs dans l'Ouest : les faibles reliefs et même les micro-reliefs qui se révèlent en lumière rasante mais surtout 
les différences de croissance qui affectent les plantes. Si le stress hydrique donne des images remarquables, cela suppose de bien connaître les caractères des végétaux, leur cycle végétatif et leurs réactions, ainsi se place en tête du palmarès le pois fourrager. Logiquement, la période la plus favorable prend place en juin, août et septembre quand se font sentir les effets de la sécheresse, mais il faut rester totalement en phase avec la météo. Il est nécessaire de repasser maintes fois sur les mêmes sites; l'heure, les cultures, un évènement météorologique particulier peuvent montrer une image remarquable mais fugace. Les bonnes surprises viennent souvent au terme d'une longue patience. Quelques renseignements techniques complètent utilement cette partie : sur les appareils photographiques, sur l'avion aussi. Ces dernières années, l'ordinateur et la photographie numérique ont considérablement bouleversé et simplifié le travail. Le cliché, tout de suite disponible, est classé sur l'ordinateur et des logiciels commodes permettent de redresser la prise de vue oblique pour obtenir un plan convenable.

Si les connaissances qui s'accumulent permettent d'identifier plus facilement les traces repérées, il demeure indispensable d'opérer des prospections à terre pour confirmer ou infirmer les interprétations. Nos trois auteurs n'hésitent pas d'ailleurs à mentionner leurs éventuelles erreurs : malgré l'expérience, il est facile de classer comme préhistorique un enclos médiéval, sans parler du fait que les sites les plus propices sont réoccupés à des époques fort éloignées.

Armé de ces indispensables renseignements, le lecteur peut alors amorcer la lecture des différents chapitres classés par ordre chronologique et dont l'importance est liée au nombre de découvertes. Chacun commence par une brève synthèse sur la période concernée, une carte localise les clichés soigneusement numérotés et, ensuite, se déroulent les photographies organisées par thèmes, chacune faisant l'objet d'un commentaire bien informé et accompagné par les références bibliographiques qui la concernent. Des croquis interprétatifs, des cartes, des photos d'objets enrichissent éventuellement le dossier. Un glossaire et surtout une précieuse bibliographie rendent d'utiles services.

Le Paléolithique est absent, à part quelques clichés aériens de grands sites reconnus depuis longtemps, Le Mont-Dol, Saulges viennent rappeler leur existence. L'archéologie aérienne commence vraiment avec le Néolithique. Entre 6000 et 2000 av. J.-C., les hommes sont bien présents dans l'Armorique et ils y laissent leur marque. Bien sûr les mégalithes, connus depuis longtemps, occupent la place attendue et l'on retrouve de très belles photos de Carnac, de Barnenez ou de Locmariaquer, ici particulièrement parlantes. Mais s'ajoute la moisson spécifique de la vision aérienne : des éperons barrés avec réseaux de talus et fossés et surtout les vastes maisons retrouvées à Pléchâtel (La Hersonnais) et à Louresse-Rochemenier (Les Choffaux).

L'âge du bronze dévoile ses enclos, souvent funéraires, ses enceintes et ses fermes et petits " villages " puis s'ouvre le gros chapitre, " Nos Ancêtres les Gaulois ", soit l'âge du fer. Là, les découvertes s'accumulent par milliers, surtout pour la seconde période, la Tène. Si l'on retrouve les clichés des oppida comme à Péaule (Morbihan), à Moulay (Mayenne) où des fouilles ont mis en évidence une agglomération, la grande découverte des archéologues aériens réside dans la révélation d'une multiplicité d'enclos d'abord plutôt ovales puis virant de plus en plus nettement à des formes quadrangulaires. Des typologies régionales semblent se préciser mais leur interprétation nécessite un gros travail, actuellement en cours. On identifie en ces formes des fermes gauloises bâties autour de la résidence du maître et entourées de cours où sont installés les bâtiments annexes, granges, greniers, silos. La densité des découvertes dans certaines zones est considérable comme dans les vallées de la Seiche ou de L'Oudon, aux limites de l'Ille-et-Vilaine et de la Mayenne, 
zones longtemps considérées comme quasi désertes. Aujourd'hui, les découvertes de G. Leroux dans une commune comme Livré-la-Touche (Mayenne) atteignent un nombre d'établissements équivalent aux exploitations actuelles. Il en va de même dans la région de Mauron en Morbihan. Les cartes compilées qui accompagnent les clichés donnent une image renouvelée de l'occupation humaine, d'autant plus que, souvent, figurent des chemins et des réseaux parcellaires.

La période romaine est plus classique mais les découvertes demeurent nombreuses. Les photographies des deux capitales de cité qui n'ont pas donné de villes par la suite : Corseul et Jublains, surtout la seconde, offrent des clichés saisissants mais la nouveauté est plus forte avec les agglomérations secondaires qui se révèlent de plus en plus. Le développement du site de l'âge du fer devenu la station routière Segora, le Fief Salmon en Maine-et-Loire, en offre l'un des meilleurs exemples avec son complexe de bâtiments civils. Le gros de l'apport vient cependant des villae disséminées dans les campagnes et que l'avion repère en quantité, même si un nombre non négligeable était déjà connu et même fouillé comme les grandes villae de la Guyomarais près de Rennes ou celle de Oisseau en Sarthe. Leur vaste espace qui s'organise autour de la pars urbana et de la pars rustica et le luxe que l'on devine en font de grands domaines qui alimentent une production agricole développée. À côté, nombre de petits établissements beaucoup plus modestes et qui prennent éventuellement la suite de fermes gauloises contribuent largement à la prospérité agricole de la région. Un intérêt particulier enfin, est porté aux voies de circulation, chemins et "voies romaines ", souvent reprises d'anciens itinéraires; la vision aérienne offre ici une contribution originale et essentielle qui permet d'étudier autant le tracé que l'emprise.

Avec "Un si long Moyen Âge ", chapitre qui n'hésite pas à brasser le millénaire médiéval, les découvertes mettent en lumière les aménagements nombreux qui marquent encore le paysage. Quelques clichés présentent les fouilles d'établissements carolingiens aristocratiques à Bressilien en Paule ou Les salles en Locronan. Pour les siècles médiévaux relativement pourvus de sources, l'archéologie aérienne peut tout autant découvrir des nouveautés qu'appuyer les textes. Elle apporte son lot de mottes avec des clichés des impressionnants édifices terroyés des Fossés Robert dans le nord de la Sarthe ou de sites beaucoup plus modestes et inconnus jusqu'ici. L'historien du paysage et du peuplement y trouve beaucoup à étudier. Les vues de terroirs comme celui de La Roë, défriché suivant des auréoles concentriques sous la direction de l'abbaye, relèvent sans doute de l'exception mais de nombreux parcellaires d'ampleur limitée créés par les paysans médiévaux apportent une contribution intéressante. Cependant, c'est dans la création du paysage rural, et en particulier du bocage, que l'apport devient incontestable. Un remarquable dossier rapproche un cliché aérien d'une page du manuscrit de la Vilaine de 1543 et d'une carte géologique, on notera aussi l'intérêt des vues de villages centrés sur leur enclos ecclésial et développés grâce à un ou des bourgs comme Ballon ou Pirmil (Sarthe). Enfin, contribution au débat, les auteurs affirment sans ambages les ruptures possibles dans les orientations des trames paysagères au cours des différentes époques.

Sans être inutiles, les chapitres suivants se révèlent moins riches mais les paysages de châteaux avec jardin et rabine montrent parfaitement l'inscription de la noblesse d'Ancien Régime dans le paysage. Enfin, l'intrusion de la modernité, après 1939, se voit accorder quelques pages qui illustrent la passion des auteurs. Archéologues, ils ont aussi plus qu'un intérêt pour le paysage. Ils y montrent à la fois le legs des guerres (blockhaus), l'intervention des grands travaux autoroutiers et ferroviaires mais, surtout, constatent et dénoncent les transformations brutales : destruction du bocage, recul des terres agricoles mangées par la modernité. 
Livre savant et de passion, Les moissons du ciel se présente comme une véritable réussite éditoriale, une somme qui deviendra un classique et pas seulement pour les historiens et archéologues de l'Ouest. Précis, documenté, présentant un choix soigneusement élaboré et mis en valeur, ce livre nous convainc d'une chose : ce trio d'aviateurs ne vit pas que dans les nuées.

Daniel PICHOT

Judic, Bruno, BecK, Robert, Bousquet-Labouerie, Christine et Lorans Élisabeth (dir.), Un Nouveau Martin. Essor et renouveaux de la figure de saint Martin IV-XXI siècle, Tours, Presses Universitaires François Rabelais, 2019 (Collection Perspectives historiques), $550 \mathrm{p}$.

Parmi les nombreuses manifestations qui, en 2016, ont marqué le $1700^{\mathrm{e}}$ anniversaire (supposé) de la naissance de saint Martin, figurait un colloque organisé à Tours sous le titre " La figure martinienne. Essor et renaissances de l'Antiquité tardive à nos jours ". Les actes de ce colloque ont été publiés en 2019 sous un titre légèrement modifié, tout à la fois plus explicite et plus ambigu, Un nouveau Martin. Essor et renouveaux de la figure de saint Martin (IVe-XXI siècle) : introduites par Bruno Judic, qui donne à voir " Les avatars de Martin " dans leur richesse et leur diversité (p. 9-34), quelques trente-trois contributions particulièrement denses fournissent la matière des trois parties de l'ouvrage (" La construction de la figure martinienne ", " Réinvestir la figure martinienne " et "Les paysages martiniens "); en guise de conclusion, Robert Beck s'applique avec minutie à analyser les enjeux idéologiques du débat suscité à la fin du XIX siècle par les publications respectives d'Albert Lecoy de la Marche et d'Ernest Babut sur l'apôtre de la Gaule (p. 519-532). Il faut déplorer que ce fort volume soit dépourvu de résumés des textes; mais, dans son introduction, Bruno Judic synthétise avec brio les différentes contributions : on se reportera donc avec profit à ce passage (p. 20-34) pour suppléer aux lacunes, aux insuffisances et aux partis pris de notre propre compte rendu. En outre, il convient de signaler que les présentations de la plupart des contributeurs ont été filmées et enregistrées lors du colloque (voir le site internet de The Hungarian Academy of Sciences [Research Center for the Humanities], en ligne à l'adresse : https://mtabtk.videotorium.hu/en/channels/2379/la-figure-martinienne [consulté le 24 mars 2020]. On peut ainsi (re)voir avec émotion l'intervention de Marianne Sághy, disparue précocement le 21 septembre 2018).

La ligne directrice étant clairement axée sur l'essor de la figure du saint et la manière dont celle-ci s'est renouvelée au cours des siècles en fonction des problématiques de chaque époque et des différents lieux où s'était développé le culte de Martin, la " matrice originelle ", c'est-à-dire l'œuvre de Sulpice Sévère, figure évidemment en bonne place : les contributions de Marianne Sághy sur "L'évêque-moine dans la Vita Martini de Sulpice Sévère " (p. 49-61) et de Xavier Gué sur "Le Christ de Sulpice Sévère : une christologie implicite dans la vita sancti Martini " (p. 85-97) s'avèrent très importantes pour la définition du "modèle de sainteté " martinien; c'est encore Sulpice Sévère qui est largement mis à contribution par Alberto Ferreiro à propos de l'aventure priscillianiste et de ses lendemains, où le rôle du saint a été minoré, voire occulté par les autres sources contemporaines, en particulier en Hispanie (p. 217-247). Les communications de Rory McTurck et de Jean-Michel Picard soulignent l'importance de la vita Martini de Sulpice Sévère comme vecteur du culte du saint, respectivement en Islande (p. 285-294) et en Irlande (p. 295-304); 